\title{
Modeling Speculative Bubbles with Diverse Investor Expectations*
}

\author{
Peter C. B. Phillips \\ Yale University, University of Auckland, \\ Singapore Management University \& University of Southampton
}

January 12, 2016

\begin{abstract}
We construct a model of asset market exuberance, collapse and recovery using subjective investor-based rational expectations about the impact of fundamentals on the market price. Investors are assumed to have heterogeneous market sentiments, allowing them to be exuberant, cautious, or fundamentalist via boundary conditions that describe their respective views of the market impact of the same economic fundamentals. Equilibrium solution paths of the model take varying forms, depending on the parameter settings that reflect the importance of each type of market participant. This rational expectations model of asset pricing is shown to be consistent with a simple explosive continuous time autoregression when exuberant sentiment dominates the market. The model explains asset price bubbles, including expansion and subsequent collapse, together with long term recovery. Extensions of the model allow for contagion effects in which market sentiments are transmitted from a primary market to a secondary market, reproducing speculative behavior and corrections in the secondary market. Some of the implications of the model for empirical work are explored.
\end{abstract}

JEL Classification: G01, G12, C32

Keywords: Asset price bubble, Boundary condition, Collapse, Contagion, Exuberance, Fundamentals, Heterogeneous agents, Recovery, Smooth pasting, Stochastic differential equation.

*The author acknowledges support of the NSF under Grant No. SES 12-58258, and helpful comments of the Editor and reviewers on the original version of this paper. 
"Stock prices are created by investors, and recognizing that investors may form different opinions even when they have the same substantive information, we contend that there can be no intrinsic value for the stock. Instead, we propose that the relevant notion of intrinsic value is obtained through market aggregation of diverse investor assessments" (Harrison and Kreps, 1978).

\section{Introduction}

The present work seeks to model asset price bubble formation and the subsequent aftermath in a manner that is well suited to econometric analysis and testing. The approach adopted draws its inspiration from Krugman's (1991) stochastic process driven rational expectations model of exchange rate target zones. This simple but highly influential model demonstrated the existence of an exchange rate equilibrium path in a currency target zone that followed an $S$ curve over a certain specified domain, thereby ensuring that the equilibrium exchange rate is maintained within a given currency band. The model formulated a single equation that related (in logarithms) the exchange rate to economic fundamentals that evolve according to a Brownian motion process, accompanied by a rational expectations term that involved the expected rate of change of the exchange rate. The solution of this equation can be represented in terms of exponential functions that are tied down locally by boundary tangency conditions (or smooth pasting by arbitrage or other considerations as in options and optimal investment theory, c.f. Dixit, 1992, Bertola, 1998) which represent mathematically the effect of countervailing actions by the monetary authority as the exchange rate nears the edges of the target band. A great advantage of this model to economic theorists and practitioners is its agnosticism with respect to the measurement of fundamentals, so that investigators may concentrate attention on the implied properties of the exchange rate rather than on structural models which seek to explain the exchange rate in terms of a (potentially long) shopping list of policy drivers and fundamentals.

The model for asset prices that we introduce here is motivated by a similar thematic. Without having to introduce a list of explicit economic fundamentals, we seek a reduced form model which encapsulates structural notions that drive stock market, commodity market, and real estate behavior and that have the capacity to explain bubble-like behavior in prices. The underlying notions may involve behavioral, game theoretic, or learning characteristics and they can be embodied in a rational expectations mechanism that accounts for subjective market assessments influencing market prices. The resulting mechanism is therefore compatible with a variety of structural notions and earlier work on modeling bubbles.

For instance, behavioral ideas of markets driven by animal spirits involving fear and greed have long existed, were presented (and the term coined) in Keynes (1936) and are now placed at centre stage in the recent work by Akerlof and Shiller (2009) that seeks to explain bubbles in financial asset and real estate 
prices in terms of such animal spirits. Earlier work by Harrison and Kreps (1978) provided an analysis of bubble behavior based on heterogeneous beliefs among investors whose market assessments aggregate to yield prices that can involve bubble characteristics when there are short sales constraints. Scheinkman and Xiong (2003) developed this notion further, providing a continuous time model of speculative bubbles driven by overconfident investors trading in a market with short sales constraints. In other recent work, Li and Xue (2009) provide a subjective Bayesian investor perspective in which "new economy thinking" acts as a driver for "belief evolution" concerning the productivity gains of new technology, leading to "rational investor" exuberance that is used to explain the 1990s Nasdaq bubble. In an alternative approach, Abreu and Brunnemeier (2003) provide a dynamic gaming explanation to justify sustained departures from fundamentals by "rational investors" riding a bubble wave even in the face of widespread recognition of inflated prices.

All of these approaches involve the use of subjective assessments about incoming economic fundamentals in investor decision making. It is this idea that is encapsulated in the model we present here. We are particularly interested in developing a reduced form model that captures a variety of structural notions such as those just described, while opening up the possibility of econometric analysis and testing. The model we develop, as will become clear, implies a discrete time version and local approximations that accord with the mildly explosive processes that have been used in recent econometric work to capture bubble-like phenomena in stock markets, commodity markets and real estate markets (see, e.g., Phillips and Yu, 2011; Phillips Shi and Yu, 2015a, 2015b). With some extension, the model can also capture cross-market contagion of speculative and negative market sentiment about the impact of fundamentals. Solutions paths reveal the effects of sentiment transmission from a primary to a secondary market, reproducing speculative bubbles with amplification or diminution depending on the extent of the contagion. Transmission effects of this type may be captured in discrete time autoregressive formulations that are suited to empirical work.

The paper is organized as follows. The next section develops the model, derives its solution, and shows how the solution is compatible with the existence of a bubble and its ultimate collapse. The conditions for bubble behavior in the sample paths are provided and some illustrations are provided. Section 3 studies the asset price dynamics arising from this model in stochastic differential equation form. The dynamics are shown to be consistent with an explosive continuous time autoregression under certain conditions on the proportion of exuberant investors and a measure of their exuberance. Section 4 extends the framework to allow for contagion effects across markets. Some empirical implications are explored in Section 5. Section 6 concludes. 


\section{Stochastic Rational Expectations and Asset Price Bubbles}

\subsection{Heterogeneous agent sentiment}

To fix ideas, we imagine a financial asset market with three heterogeneous types of investors that we call fundamentalists, exuberants and circumspects. These investors all look at the same incoming (log) fundamentals $\left(f_{t}\right)$ but interpret them in different ways thinking that the market will follow, exaggerate or diminish their impact. In their financial market actions, we may regard such investors as responsive (making proportionate responses to changes in fundamentals), over-responsive (exuberant when fundamentals increase and gloomy when fundamentals decline), or underresponsive (cautious and reacting more slowly when fundamentals change). Cautious investors tend to reduce overall variation and overreponsive investors tend to raise volatility by exaggerating fundamental effects.

This classification of investor type simplifies the reality of a much more general (and evolving) distribution of market participants covering a continuous spectrum of bull, bear, and conservative sentiment. The market price is affected by economic fundamentals in conjunction with the interplay of the various types of investors and their differing subjective view about the impact of the market fundamentals on asset prices over some forthcoming horizon. The structural process by which this interplay occurs, via brokerage orders, limit orders, or mutual fund and hedge fund operations is not specified. Instead, we provide the market price to be determined by a probabilistic mechanism that embodies the outcome of investor actions and sentiment which is expressed through a subjective, speculative, rational expectations component. The mechanism is explained as follows.

We suppose there are three classes of investors - exuberant $(a)$, cautious $(b)$, and fundamentalist $(c)$ - signified by these subscripts and with respective proportions in the population given by $p_{a}, p_{b}$, and $p_{c}$ satisfying $p_{a}+p_{b}+p_{c}=1$. Each class implements an investment strategy based on subjective response functions to the same fundamentals. The reactions may also depend on (typically) unobserved extraneous data that these investors introduce to their decision making and market positions. Fundamentals are also typically unobserved (to the econometrician) and summarize a vast amount of incoming information, assumed to be embodied in a scalar stochastic process index that represents fundamental value $f_{t}$. We assume that the market $(\log )$ price response $\left(s_{t}\right)$ to fundamentals is influenced by the sentiments and strategies of these investors according to their respective proportions in the market leading to outcomes $s_{i t}, i=a, b, c$. For our initial development, these proportions are taken as fixed, but may also be considered time dependent and/or responsive to market conditions, as will be pursued later. The collective market response function then takes the form of the weighted average of these outcomes, viz.,

$$
s_{t}=p_{a} s_{a t}+p_{b} s_{b t}+p_{c} s_{c t}=p_{c} f_{t}+p_{a} s_{a t}+p_{b} s_{b t},
$$


which uses the fact that $s_{c t}=f_{t}$ because the fundamentalist strategy follows fundamentals exactly. The aggregation involved in yielding the market price (1) reflects the notion emphasized by Harrison and Kreps (1978) cited in the header that "intrinsic value is obtained through market aggregation of diverse investor assessments."

The behavior of exuberant and cautious investors takes account of subjective "rational expectations" of the impact of fundamentals on the market price. Analogous to Krugman's (1991) exchange rate model, we write

$$
s_{i t}=f_{t}+\gamma_{i} \mathbb{E}_{i t}\left(d s_{i t}\right) / d t, i=a, b,
$$

where $\mathbb{E}_{i t}(i=a, b)$ is investor $i$ expectation given information up to time $t$, including fundamentals $f_{t}$. The term $\mathbb{E}_{i t}\left(d s_{i t}\right) / d t$ represents investor $i$ 's expected rate of change of the stock price and $s_{i t}=f_{t}+\gamma_{i} \mathbb{E}_{i t}\left(d s_{i t}\right) / d t$ is the overall response to common fundamentals $f_{t}$ that is expected by investors in this class. These expectations are influenced by investor sentiment about how prices will be affected by a change in fundamentals, with investor $a$ anticipating a more aggressive response than investor $b$. Combining these effects we have the collective market response

$$
\begin{aligned}
s_{t} & =p_{c} f_{t}+\sum_{i=a, b} p_{i}\left\{f_{t}+\gamma_{i} \mathbb{E}_{i t}\left(d s_{i t}\right) / d t\right\} \\
& =f_{t}+\sum_{i=a, b} p_{i} \gamma_{i} \mathbb{E}_{i t}\left(d s_{i t}\right) / d t
\end{aligned}
$$

which depends on investor expectations and the proportion of investors in each class.

\subsection{Model solution}

While each component $s_{i t}$ follows (2), the solution turns out to be different from the Krugman $S$ curve, where fundamentals are attentuated by tangency conditions to keep the exchange rate within a fixed band at least within a local region of fundamentals. In the present case, exuberant investors $(a)$ anticipate a stronger response of prices to fundamentals, an effect which generates stronger growth in the presence of favorable economic conditions and greater volatility (and risk) when conditions turn unfavorable. The framework is therefore hierarchical with each submodel generating its own solution according to its own boundary conditions that characterize the investor sentiment.

The model for exuberant investors $s_{a t}=f_{t}+\gamma_{a} \mathbb{E}_{a t}\left(d s_{a t}\right) / d t$ is solved as in Krugman (1991) using a trial solution $s_{a}=s_{a}(f)=f+A_{a}\left(e^{\alpha_{a} f}-e^{-\alpha_{a} f}\right)$. The model differs from the Krugman model in the way we incorporate investor sentiment through first order boundary conditions. To characterize exuberance, the right derivative of $s=s(f)$ is assumed to satisfy $s_{a}^{\prime}(0+)=1+\lambda_{a}>1$, where $\lambda_{a}>0$ is a measure of the market exuberance of the investor at the origin initial condition $f=0$ in response to a positive infinitesimal change in 
fundamentals $f>0$. This boundary condition implies an accentuated effect of (positive) fundamentals on asset prices relative to a normal market reaction that directly responds to fundamentals through the equation $s_{t}=f_{t}$ that accords with fundamentals and has unit slope. In doing so, this boundary condition also differs from Krugman's smooth pasting tangency condition, which requires that $s^{\prime}\left(f_{*}\right)=0$ at some designated boundary points $\pm f_{*}$ (determined by the upper and lower limits of the exchange rate target zone). Importantly when the Krugman boundary conditions $s^{\prime}\left(f_{*}\right)=s^{\prime}\left(-f_{*}\right)=0$ are imposed, the Krugman solution is well determined within the region $\left[-f_{*}, f_{*}\right]$ whereas outside this (local) region of the origin the solution will inevitably diverge in view of the exponential form of the components of $s(f)$.

In the present case, upon differentiation of the trial solution, the boundary condition $s_{a}^{\prime}(0+)=1+\lambda_{a}$ yields $A_{a}=\lambda_{a} /\left(2 \alpha_{a}\right)>0$, and substitution gives

$$
s_{a}(f)=f+\frac{\lambda_{a}}{2 \alpha_{a}}\left(e^{\alpha_{a} f}-e^{-\alpha_{a} f}\right), \text { with } \alpha_{a}=\sqrt{\frac{2}{\gamma_{a} \sigma^{2}}},
$$

for $f \geq 0$. The parametric solution $\alpha_{a}=\sqrt{2 /\left(\gamma_{a} \sigma^{2}\right)}$ is obtained as follows. Assuming $f_{t}$ is Brownian motion with variance $\sigma^{2}$ and using the stochastic differential $d s_{a}=s_{a}^{\prime} d f+\frac{\sigma^{2}}{2} s_{a}^{\prime \prime}(f) d t$, we deduce that

$$
\mathbb{E}_{a t}\left(d s_{a}\right) / d t=\frac{\sigma^{2}}{2} s_{a}^{\prime \prime}(f)=\frac{\alpha_{a} \sigma^{2}}{4} \lambda_{a}\left(e^{\alpha_{a} f}-e^{-\alpha_{a} f}\right),
$$

which in conjunction with the equation $s_{a t}=f_{t}+\gamma_{a} \mathbb{E}_{a t}\left(d s_{a t}\right) / d t$ and (4) implies that $\alpha_{a}=\sqrt{2 /\left(\gamma_{a} \sigma^{2}\right)}$, a result that corresponds to Krugman's (1991) parametric solution for the exponent $\alpha_{a}$ in terms of the structural parameters $\left(\gamma_{a}, \sigma^{2}\right)$ in the exchange rate model.

A similar approach leads to the solution for $s_{b}(f)$. In this case we characterize cautious investors by the requirement that the first derivative satisfies the boundary condition $s_{b}^{\prime}(0+)=1-\lambda_{b}<1$, where $\lambda_{b} \in(0,1)$ is a measure of the cautious market sentiment exhibited by the circumspect investor at $f=0$ in response to a positive infinitesimal change in fundamentals. This boundary condition attenuates the effect of fundamentals at the origin by delivering a slope less than unity, which corresponds to the normal market reaction to fundamentals in which $s_{a t}=f_{t}$ has martingale behavior. Proceeding as before we obtain the solution

$$
s_{b}(f)=f-\frac{\lambda_{b}}{2 \alpha_{b}}\left(e^{\alpha_{b} f}-e^{-\alpha_{b} f}\right), \text { with } \alpha_{b}=\sqrt{\frac{2}{\gamma_{b} \sigma^{2}}} .
$$

Combining (4) and (5) in (3) we have, for $f_{t} \geq 0$,

$$
\begin{aligned}
s_{t} & =f_{t}+p_{a} \frac{\alpha_{a} \gamma_{a} \lambda_{a} \sigma^{2}}{4}\left(e^{\alpha_{a} f_{t}}-e^{-\alpha_{a} f_{t}}\right)-p_{b} \frac{\alpha_{b} \gamma_{b} \lambda_{b} \sigma^{2}}{4}\left(e^{\alpha_{b} f_{t}}-e_{t}^{-\alpha_{b} f_{t}}\right) \\
& =f_{t}+\frac{p_{a} \lambda_{a}}{\alpha_{a}} \sinh \left(\alpha_{a} f_{t}\right)-\frac{p_{b} \lambda_{b}}{\alpha_{b}} \sinh \left(\alpha_{b} f_{t}\right) .
\end{aligned}
$$


Noting the form $\alpha=\sqrt{\frac{2}{\gamma \sigma^{2}}}$, we see that larger volatility (higher $\sigma^{2}$ ) reduces $\alpha$ in both cases and therefore attenuates the exponential path and therefore divergence from fundamentals. An extension of the model that allows for greater flexibility is obtained by introducing a Bayesian prior perception of the volatility in fundamentals. This is achieved by permitting the prior view of $\sigma^{2}$ to differ for $i=a, b$. Then, perception of higher risk with greater $\sigma_{a}^{2}$ would lead to a reduction in the exponential path, thereby attenuating exuberant investor effects. Likewise, the perception of lower risk with smaller $\sigma_{b}^{2}$ would lead to a stronger exponential solution path that accentuates cautious investor effects on the outcome and equilibrium path.

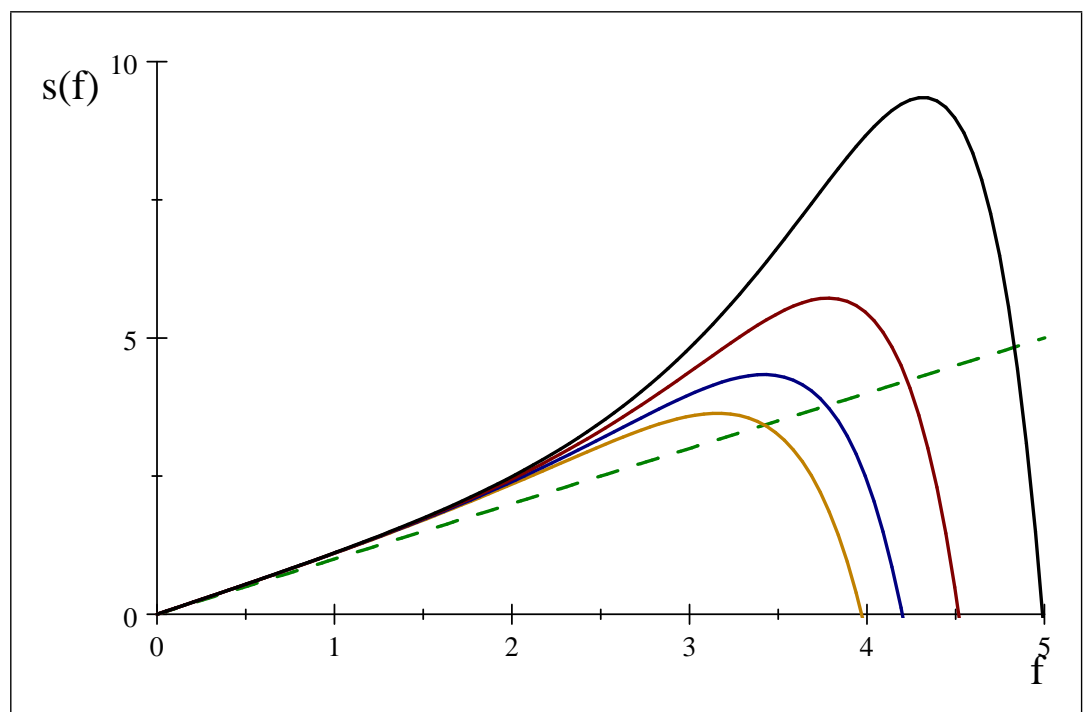

Figure 1a: Solution paths $s(f)$ for equilibrium asset prices with parameter settings $\alpha_{a} \in\{1.62,1.64,1.66,1.68\}$ shown in sienna, blue, red, and black, and using common settings for the other parameters, as given in the text. The curves are plotted against the $45^{\circ}$ line (green dashed) where $s=f$.

As is also apparent from the exponential component form of (6), the solution $s_{t}$ ultimately diverges as $f_{t} \rightarrow \infty$, just as the exhange rate solution in the Krugman model does outside the smooth pasting limits at $\pm f_{*}$. In the case of Figure 1a, where $\alpha_{b}>\alpha_{a}$ and negative investor sentiment ultimately dominates, the solution for the log price $s_{t}$ eventually diverges according as $s_{t} \sim-\frac{p_{b} \lambda_{b}}{\alpha_{b}} \sinh \left(\alpha_{b} f_{t}\right) \sim-p_{b} \frac{\alpha_{b} \gamma_{b} \lambda_{b} \sigma^{2}}{4} e^{\alpha_{b} f_{t}} \rightarrow-\infty$ as $f_{t} \rightarrow \infty$. Thus, (6) may be interpreted as a local solution for $s_{t}$ under positive fundamentals ${ }^{1}$.

An analogous local solution for $s_{t}$ holds for negative fundamentals under response conditions at the origin where $s_{a}^{\prime}(0-)=1-\lambda_{a}<1$ (so that exuberant investors take a more optimistic view of the impact of negative fun-

\footnotetext{
${ }^{1}$ Correspondingly, the Krugman model provides a local solution for the exchange rate within the domain $\left[-f_{*}, f_{*}\right]$ as the boundary conditions imposed by the model do not prevent divergence outside of this domain.
} 
damentals on price) and $s_{b}^{\prime}(0-)=1+\lambda_{b}>1$ (so that cautious investors take a more pessimistic view of negative fundamentals on price). In this alternative scenario, pessimistic sentiment again eventually dominates and $s_{t} \sim$ $-p_{b} \frac{\alpha_{b} \gamma_{b} \lambda_{b} \sigma^{2}}{4} e^{-\alpha_{b} f_{t}} \rightarrow-\infty$ as $f_{t} \rightarrow-\infty$ when $\alpha_{b}>\alpha_{a}$. The combined solution can therefore be written in the following form

$$
\begin{aligned}
s_{t}= & f_{t}+\left\{\frac{p_{a} \lambda_{a}}{\alpha_{a}} \sinh \left(\alpha_{a} f_{t}\right)-\frac{p_{b} \lambda_{b}}{\alpha_{b}} \sinh \left(\alpha_{b} f_{t}\right)\right\} 1\left\{f_{t} \geq 0\right\} \\
& +\left\{-\frac{p_{a} \lambda_{a}}{\alpha_{a}} \sinh \left(\alpha_{a} f_{t}\right)+\frac{p_{b} \lambda_{b}}{\alpha_{b}} \sinh \left(\alpha_{b} f_{t}\right)\right\} 1\left\{f_{t}<0\right\} .
\end{aligned}
$$

Figure 1b displays the two-sided solution paths consonant with such parameter configurations that allow for positive and negative departures of fundamentals from the origin with ultimate dominance of negative investor sentiment along both paths.

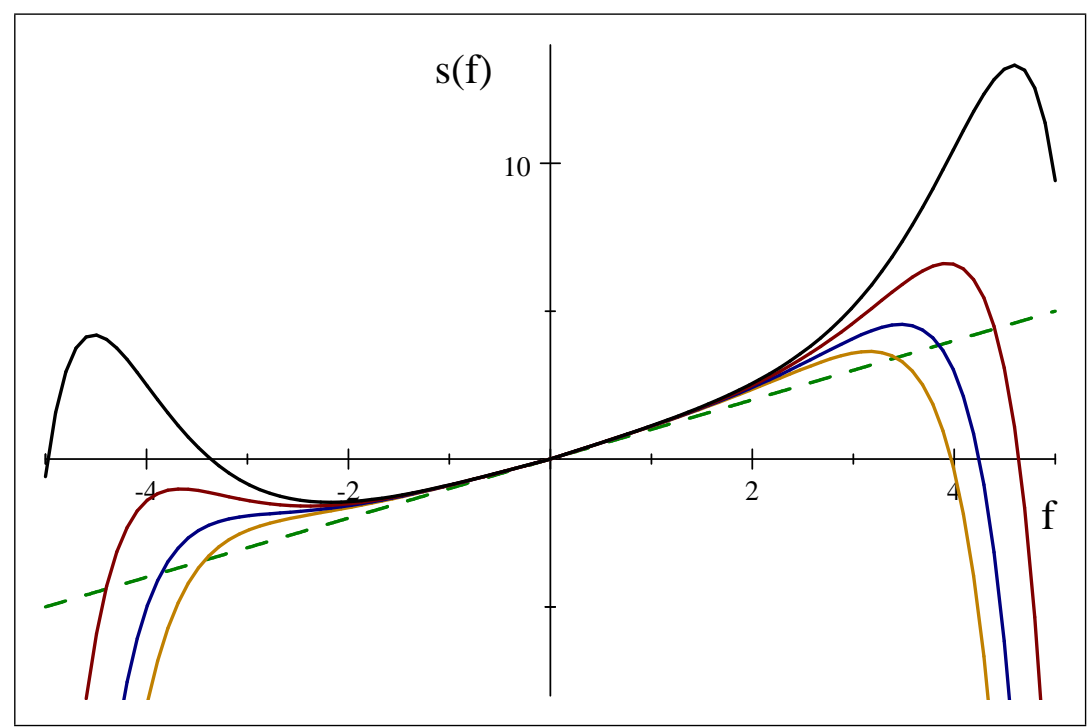

Figure 1b: Two sided solution paths $s(f)$ for equilibrium asset prices with parameter settings $\alpha_{a} \in\{1.62,1.64,1.66,1.68\}$ shown in sienna, blue, red, and

black, and using common settings for the other parameters, as given in the text. The curves are plotted against the $45^{\circ}$ line (green dashed) where $s=f$.

\subsection{Illustrations}

As is immediately apparent from (6), when the boundary effects cancel $\left(\lambda_{a}=\lambda_{b}\right)$ and the scale effects and proportions are both equal $\left(\gamma_{a}=\gamma_{b}\right.$; and $\left.p_{a}=p_{b}\right)$, then $s=f$ and the solution path for $s$ follows fundamentals. Disparities in these parameters produce divergences from the fundamental path and these take various forms. Figures $1 \mathrm{a}$ and $1 \mathrm{~b}$ show the solution paths $s=s(f)$ for various values of the parameters $\left\{\lambda_{a}, \lambda_{b}, p_{a}, p_{b}, \alpha_{a}, \alpha_{a}\right\}$ against the efficient market martingale 
model in which prices simply follow Brownian motion fundamentals along the $45^{\circ}$ line $s=f$.

In these figures we set $p_{a}=0.5, p_{b}=0.3, p_{c}=1-p_{a}-p_{b}=0.2 ; \lambda_{a}=0.2$, $\lambda_{b}=0.2 ; \alpha_{a} \in\{1.62,1.64,1.66,1.68\}$, and $\alpha_{b}=1.8$. The difference in the probability weights $\left(p_{a}, p_{b}\right)$ and the range of values used for $\alpha_{a}=\sqrt{2 / \gamma_{a} \sigma^{2}}$ show how the strength of exuberance in relation to circumspection in investing plays a large role in the shape of the market response to movements in fundamentals. As is apparent in Figures $1 \mathrm{a}$ and $1 \mathrm{~b}$, the influence of exuberant investment dominates $s_{t}$ initially so that $s(f)>f$ and prices exceed fundamentals until the point of intersection $s(f)=f$, a value that is dependent on the particular parameter configuration $\left(p_{a}, p_{b}, \lambda_{a}, \lambda_{b}, \alpha_{a}, \alpha_{b}\right)$. Cases where $a_{a}$ is larger clearly exaggerate the differential between $s$ and $f$. This dominance occurs even though in the above example the exponent $\alpha_{a}<\alpha_{b}$ and is explained by the fact that the proportion of exuberant investors, $p_{a}=0.5$, exceeds the proportion of cautious investors, $p_{b}=0.3$. However, since $\alpha_{b}>\alpha_{a}$, the influence of the circumspect investors eventually takes over and the bubble collapses. The peak of the bubble $s(\bar{f})$ when $f>0$ occurs at the stationary point $\bar{f}$ which satisfies

$$
s^{\prime}(\bar{f})=1+p_{a} \lambda_{a} \cosh \left(\alpha_{a} \bar{f}\right)-p_{b} \lambda_{b} \cosh \left(\alpha_{b} \bar{f}\right)=0 .
$$

The equation (7) can be solved numerically ${ }^{2}$, giving $\bar{f}=3.32,3.55,3.87,4.36$ when $\alpha_{a}=1.62,1.64,1.66,1.68$. These values of $\bar{f}$ match those corresponding to the peaks of the curves shown in Figure 1a.

Further differentiation yields $\partial s^{\prime}(f) / \partial \alpha_{a}=p_{a} \lambda_{a} \alpha_{a} \sinh \left(\alpha_{a} f\right)>0$ and $\partial s^{\prime}(f) / \partial \alpha_{b}=-p_{b} \lambda_{b} \alpha_{b} \sinh \left(\alpha_{b} f\right)<0$, so that the slope of the solution path $s(f)$ increases with $\alpha_{a}$ and decreases as $\alpha_{b}$ increases. Thus, speculative bubble effects become stronger as the exuberance exponent $\alpha_{a}$ rises and become weaker with increases in $\alpha_{b}$. It is apparent from the shape of the curves in Figure 1a that the change from ascent to descent around the peak of the bubble appears most rapid in the case where $\alpha_{a}$ takes the largest value and the peak differential over fundamentals is the greatest. In this case, the derivative $s^{\prime}(f)$ changes rapidly from the largest exponential rate $\left(\alpha_{a}=1.68\right)$ of increase during the bubble expansion to the descent rate $\left(\alpha_{b}=1.80\right)$ that is common for the other cases.

\footnotetext{
${ }^{2}$ An approximate solution of (7) is obtained by factoring out the dominant exponential and using a logarithmic expansion to simplify the equation and obtain an approximate analytic solution. This has the form

$$
\bar{f} \simeq \frac{\ln \left(\frac{p_{a} \lambda_{a}}{p_{b} \lambda_{b}}\right)}{\alpha_{b}-\alpha_{a}}+\frac{2}{p_{a} \lambda_{a}\left(\alpha_{b}-\alpha_{a}\right)}\left(\frac{p_{b} \lambda_{b}}{p_{a} \lambda_{a}}\right)^{\alpha_{a}\left(\alpha_{b}-\alpha_{a}\right)},
$$

when $\bar{f}$ is large. The first term of (8) yields the approximate values $\bar{f}=2.55(3.32), 2.84$ (3.55), 3.65 (3.87), 4.26 (4.36) with true values in parentheses, corresponding to the cases $\alpha_{a}=1.62,1.64,1.66,1.68$.
} 


\subsection{Modeling long-run market recovery}

We may superpose on the above model a decay system that achieves long-run recovery returning the market to fundamental values. The mechanism inhibits market exuberance at peak levels and controls negativism following a downturn. The mechanism operates through the market proportions of exuberant and cautious investors. Instead of fixing these proportions as parameters, we allow them to be ex post dependent on fundamentals using the functional forms $p_{a}=p_{a} e^{-\delta_{a} f_{t}^{2}}$ and $p_{b}=p_{b} e^{-\delta_{b} f_{t}^{2}}$ with $\delta_{a}, \delta_{b} \geq 0$, so that the proportions of exuberant and cautious investors decay to zero eventually as $f \rightarrow \pm \infty$, bringing the model back to fundamental values. ${ }^{3}$ The modified model incorporating the decay feature has the following form for $f_{t} \geq 0$

$$
s_{t}=f_{t}+\frac{p_{a} \lambda_{a} e^{-\delta_{a} f_{t}^{2}}}{\alpha_{a}} \sinh \left(\alpha_{a} f_{t}\right)-\frac{p_{b} \lambda_{b} e^{-\delta_{b} f_{t}^{2}}}{\alpha_{b}} \sinh \left(\alpha_{b} f_{t}\right) .
$$

With some adjustments to the decay mechanism such as the use of an endogenous response like $e^{-\delta\left(s_{t}-f_{t}\right)^{2}}$ in place of $e^{-\delta f_{t}^{2}}$, the mechanism may be associated with policy intervention. For instance, to control housing market exuberance in relation to fundamentals, regulators may resort to macro-prudential measures such as capping loan to value ratios, imposing or increasing stamp duties, and reducing debt servicing ratios. These measures may be introduced over a period of time with the goal of attenuating market exuberance. Their effects are intended to take heat out of the property market and promote long term market recovery. Recovery is accomplished by diminishing overall investor exuberance. In the present context, the mechanism works by reducing the proportion $p_{a}$, the exuberance measure $\lambda_{a}$, and counteracting the exponential growth effects in the solution. As such policy intervention occurs, cautious investors gain in confidence that the market will better reflect fundamental values, which leads to a corresponding attenuation of the effects of negative sentiment.

A recent example of such sustained policy intervention in the property market is the series of ten successive rounds of macro-prudential cooling measures implemented by regulatory authorities in Singapore over the period 2009 - 2013. Using bubble detection techniques based on the estimation of a reduced form autoregression and the use of right-sided unit root tests for explosive behavior, Liang et al (2016) found that these measures appear to have been effective in containing exuberance in Singapore's private housing market.

The decay mechanism in (9) may also be interpreted as a transversality condition which ensures that the market price returns to fundamentals in the long run as fundamental values evolve and local departures from fundamentals are eliminated as the proportions of optimistic and pessimistic investors declines. Transversality conditions have been used in past work to exclude the occurrence of bubbles in asset pricing models by completely eliminating all solutions that include bubbles (e.g., see Becker, 2008). In (9), the transversality decay mechanism does not prevent the emergence of bubbles and crashes in finite horizon

${ }^{3}$ This formulation may be interpreted as time dependent changes in the proportions $\left(p_{a}, p_{b}\right)$ under the assumption that fundamentals $f$ eventually diverge with the passage of time. 
(local) solutions but its imposition ensures that fundamental values are followed eventually.

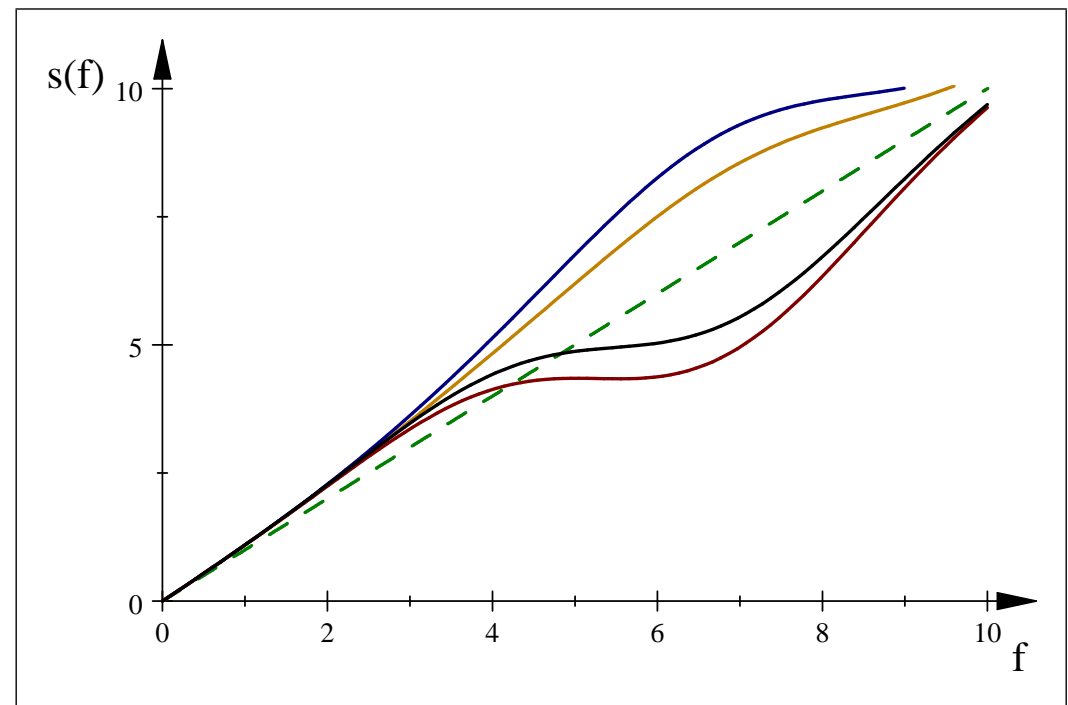

Figure 2: Solution paths for equilibrium asset prices that follow the same settings and schematic as Figure 1a, but now allow for evolutionary decay in the proportions of exuberant and circumspect investors with $p_{a}(f)=e^{-\delta_{a} f^{2}}$ and $p_{b}(f)=e^{-\delta_{b} f^{2}}$. The curves show both decay in exuberance (sienna and blue with settings $\delta_{a}=0.16, \delta_{b}=0.14$ ) and decay in negativism (red and black with equal settings $\delta_{a}=\delta_{b}=0.15$ ).

Figure 2 shows the impact of this decay system on the solution paths given in Figure 1a. The solution paths use the same settings and color schematics as those given in Figure 1a but introduce evolutionary decay in the proportions according to $p_{a}(f)=p_{a} e^{-\delta_{a} f^{2}}$ and $p_{b}(f)=p_{b} e^{-\delta_{b} f^{2}}$. The curves show both decay in exuberance (the sienna and blue curves with settings $\delta_{a}=0.14$ and $\delta_{b}=0.16$ ) and decay in negativism (red and black with equal settings $\delta_{a}=\delta_{b}=$ $0.15)$.

Various refined specifications are possible. For instance, the proportions could be made time dependent directly, so that $p_{a}=p_{a}(t)$ and $p_{b}=p_{b}(t)$, a specification that embodies exogenous forcing of the investor proportions and may reflect the influence of unspecified and unobserved driver variables. The price path would then have the form $s_{t}=s\left(f_{t}, t ; \theta\right)$ and stochastic differentiation of $s_{t}$ would correspondingly involve further terms arising from direct time differentiation. The specification (9) is a simplification that ensures ex post that the effects of evolution in the proportion of exuberant and circumspect investors are directly embodied in the reduced form. The decay system acts like a transversality condition that brings the model back to fundamentals eventually, while not preventing intermediate episodes of exuberance and collapse. While the exponential formulation of the decay system is arbitrary, it is necessary to 
use some form of (higher order) exponential decay to ensure that the exponential effects involved in the episodes of exuberance and collapse are eventually moderated and there is no permanent divergence from fundamentals.

In a structural model, investors would recognize that departures from fundamentals are unlikely to continue indefinitely (in view of the presence of such long term market effects, arbitrage, or policy intervention) and that the opportunities for more aggressive investing than may be warranted by fundamentals are inevitably finite horizon (subjectively apparent) opportunities. Investment decisions based on present value calculations would then need to take into account the implied finite horizon framework. Lee and Phillips (2016) provide some analysis of investment time horizons that acommodate myopic investing and the impact of speculative behavior that focuses on short run market gains over the long run effects of fundamentals.

\section{Asset Price Dynamics}

The solution (6) delivers asset price dynamics for $s_{t}$ by stochastic differentiation. These dynamics are of interest because they help guide empirical specifications. We simplify notation by introducing the parameter vector $\theta=$ $\left(p_{a}, p_{b}, \lambda_{a}, \lambda_{b}, \alpha_{a}, \alpha_{b}, \sigma^{2}\right)$. Then, differentiation of the solution $s_{t}\left(f_{t} ; \theta\right)$ yields the nonlinear stochastic differential equation

$$
d s_{t}=\mu\left(f_{t} ; \theta\right) d t+\sigma\left(f_{t} ; \theta\right) d f_{t},
$$

with instantaneous drift given by

$$
\mu\left(f_{t} ; \theta\right)=\frac{\sigma^{2}}{2}\left\{p_{a} \lambda_{a} \alpha_{a} \sinh \left(\alpha_{a} f_{t}\right)-p_{b} \lambda_{b} \alpha_{b} \sinh \left(\alpha_{b} f_{t}\right)\right\},
$$

and instantaneous volatility

$$
\sigma\left(f_{t} ; \theta\right)=1+p_{a} \lambda_{a} \cosh \left(\alpha_{a} f_{t}\right)-p_{b} \lambda_{b} \cosh \left(\alpha_{b} f_{t}\right) .
$$

\subsection{Local behavior after initialization}

The properties of the diffusion (10) can be investigated locally in the neighborhood of certain points. For instance, in the vicinity of the origin $f_{t} \sim 0$, we may use the power series representation of the hyperbolic functions to give local approximations to $\mu\left(f_{t} ; \theta\right)$ and $\sigma\left(f_{t} ; \theta\right)$. We obtain

$$
\mu\left(f_{t} ; \theta\right)=\frac{\sigma^{2}}{2}\left\{p_{a} \lambda_{a} \alpha_{a}^{2}-p_{b} \lambda_{b} \alpha_{b}^{2}\right\} f_{t}+O\left(f_{t}^{3}\right) \simeq \frac{\sigma^{2}}{2}\left\{\frac{p_{a} \lambda_{a} \alpha_{a}^{2}-p_{b} \lambda_{b} \alpha_{b}^{2}}{1+p_{a} \lambda_{a}-p_{b} \lambda_{b}}\right\} s_{t},
$$

since $s_{t}=f_{t}+\frac{p_{a} \lambda_{a}}{\alpha_{a}} \sinh \left(\alpha_{a} f_{t}\right)-\frac{p_{b} \lambda_{b}}{\alpha_{b}} \sinh \left(\alpha_{b} f_{t}\right)=\left(1+p_{a} \lambda_{a}-p_{b} \lambda_{b}\right) f_{t}$ for $f_{t} \sim 0$. Also

$\sigma\left(f_{t} ; \theta\right)=1+p_{a} \lambda_{a} \cosh \left(\alpha_{a} f_{t}\right)-p_{b} \lambda_{b} \cosh \left(\alpha_{b} f_{t}\right)=\left(1+p_{a} \lambda_{a}-p_{b} \lambda_{b}\right)+O\left(f_{t}^{2}\right)$. 
Combining (11) and (12) we can approximate (10) by the first order stochastic differential equation

$$
d s_{t}=\beta s_{t} d t+\omega d f_{t}
$$

where

$$
\beta=\frac{\sigma^{2}}{2}\left\{\frac{p_{a} \lambda_{a} \alpha_{a}^{2}-p_{b} \lambda_{b} \alpha_{b}^{2}}{1+p_{a} \lambda_{a}-p_{b} \lambda_{b}}\right\} \text { and } \omega=1+p_{a} \lambda_{a}-p_{b} \lambda_{b} .
$$

Hence, in the vicinity $f_{t} \sim 0, s_{t}$ follows an Ornstein-Uhlenbeck (OU) process with constant coefficients $\beta=\frac{\sigma^{2}}{2}\left\{p_{a} \lambda_{a} \alpha_{a}^{2}-p_{b} \lambda_{b} \alpha_{b}^{2}\right\} / \omega$ and $\omega=1+p_{a} \lambda_{a}-p_{b} \lambda_{b}$. When $\beta>0$, it is clear that the model (13) is explosive and, correspondingly, the solution process $s_{t}$ is locally explosive in the vicinity of the origin. Observe that this condition for an explosive model $(\beta>0)$ is satisfied when the model parameters satisfy $\alpha_{a}^{2}>\frac{p_{b} \lambda_{b}}{p_{a} \lambda_{a}} \alpha_{b}^{2}$. It follows that explosive behavior may arise

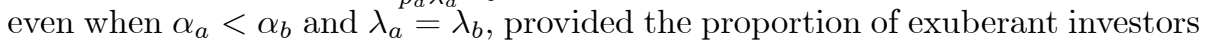
$\left(p_{a}\right)$ is sufficiently great relative to the proportion of circumspect investors $\left(p_{b}\right)$. Such a configuration arises in the example of Figure 1a, which shows how explosive behavior may dominate negative sentiment initially, even though stronger negative exponential effects manifest through the term $\sinh \left(\alpha_{b} f_{t}\right)$ in the solution. These negative effects eventually become dominant for large $f_{t}$ producing bubble collapse, as is clearly evident in Figure 1a.

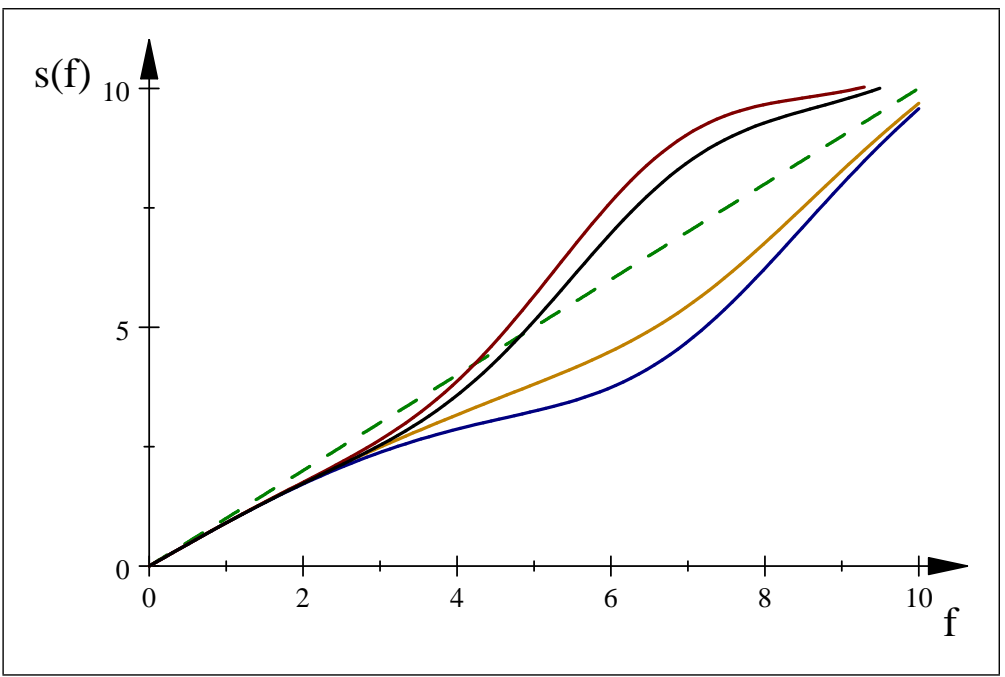

Figure 3: Solution paths for equilibrium asset prices with the reverse settings of Figure 1a (see footnote 3) and with evolutionary decay in the proportions of exuberant and circumspect investors with $p_{a}(f)=e^{-\delta_{a} f^{2}}$ and $p_{b}(f)=e^{-\delta_{b} f^{2}}$. The curves show both the erosion of negativism (sienna and blue with settings $\delta_{a}=0.16, \delta_{b}=0.14$ ) and decay in subsequent exuberance (red and black with equal settings $\delta_{a}=\delta_{b}=0.15$ ).

Explosive behavior also manifests when the market exuberance measure $\lambda_{a}$ is large enough relative to the market caution measure $\lambda_{b}$ even in situations 
where the proportion of exuberant investors may not be as great as that of circumspect investors (i.e., $p_{a} \leq p_{b}$ ) provided the condition $p_{a} \lambda_{a} \alpha_{a}^{2}>p_{b} \lambda_{b} \alpha_{b}^{2}$ still holds. In short, the subjective rational expectations model of asset pricing is consistent with a simple explosive continuous time autoregression when exuberant sentiment dominants the market either by the proportion $\left(p_{a}\right)$ of such investors or the strength of their market positions $\left(\lambda_{a}\right.$ and $\left.a_{a}\right)$.

Next consider the case where $p_{a} \lambda_{a} \alpha_{a}^{2}<p_{b} \lambda_{b} \alpha_{b}^{2}$ and the coefficient $\beta<0$ in (13). This case arises when cautious investors dominate the market initially due to the size of $p_{b}$ and $\lambda_{b}$ relative to $p_{a}$ and $\lambda_{a}$. Their behavior leads to a diminished response of market prices to fundamentals. The process $s_{t}$ then follows a stationary Ornstein-Uhlenbeck process which produces a market correction. As this correction continues, it is viewed by exuberant investors as a buying opportunity in which fundamental values exceed market prices. Ultimately, the sustained bear market is overtaken by exuberance among these investors and a new bubble emerges, due to the fact that $\alpha_{a}>\alpha_{b}$. Figure 3 gives an illustration of such a market correction and recovery using parametric settings that are the reverse to those shown in Figure 2 with the same decay effects active. ${ }^{4}$

\subsection{Peak and trough behavior}

In the vicinity of the peak value $\bar{f}$ that satisfies (7) we have the following expansions of the drift and volatility functions

$\mu\left(f_{t} ; \theta\right)=\mu(\bar{f} ; \theta)+\frac{\sigma^{2}}{2}\left\{p_{a} \lambda_{a} \alpha_{a}^{2} \cosh \left(\alpha_{a} \bar{f}\right)-p_{b} \lambda_{b} \alpha_{b}^{2} \cosh \left(\alpha_{b} \bar{f}\right)\right\} d f_{t}+O_{p}(d t)$,

and

$$
\begin{aligned}
\sigma\left(f_{t} ; \theta\right) & =\sigma(\bar{f} ; \theta)+\left\{p_{a} \lambda_{a} \alpha_{a} \sinh \left(\alpha_{a} \bar{f}\right)-p_{b} \lambda_{b} \alpha_{b} \sinh \left(\alpha_{b} \bar{f}\right)\right\} d f_{t}+O_{p}(d t) \\
& =\left\{p_{a} \lambda_{a} \alpha_{a} \sinh \left(\alpha_{a} \bar{f}\right)-p_{b} \lambda_{b} \alpha_{b} \sinh \left(\alpha_{b} \bar{f}\right)\right\} d f_{t}+O_{p}(d t),
\end{aligned}
$$

since $\sigma(\bar{f} ; \theta)=1+p_{a} \lambda_{a} \cosh \left(\alpha_{a} \bar{f}\right)-p_{b} \lambda_{b} \cosh \left(\alpha_{b} \bar{f}\right)=0$ at the peak value $\bar{f}$ in view of (7). Combining these representations in (10), we find that the local change of $s_{t}$ at $f_{t} \sim \bar{f}$ is given by

$$
\begin{aligned}
d s_{t} & =\mu(\bar{f} ; \theta) d t+\left[\sigma(\bar{f} ; \theta)+\left\{p_{a} \lambda_{a} \alpha_{a} \sinh \left(\alpha_{a} \bar{f}\right)-p_{b} \lambda_{b} \alpha_{b} \sinh \left(\alpha_{b} \bar{f}\right)\right\} d f_{t}\right] d f_{t}+o_{p}(d t) \\
& =\mu(\bar{f} ; \theta) d t+\left\{p_{a} \lambda_{a} \alpha_{a} \sinh \left(\alpha_{a} \bar{f}\right)-p_{b} \lambda_{b} \alpha_{b} \sinh \left(\alpha_{b} \bar{f}\right)\right\} \sigma^{2} d t+o_{p}(d t) \\
& =\frac{3 \sigma^{2}}{2}\left\{p_{a} \lambda_{a} \alpha_{a} \sinh \left(\alpha_{a} \bar{f}\right)-p_{b} \lambda_{b} \alpha_{b} \sinh \left(\alpha_{b} \bar{f}\right)\right\} d t .
\end{aligned}
$$

Although the equilibrium path $s=s(f)$ has a turning point at $f=\bar{f}$, this fact does not imply that the stochastic process $s_{t}=s\left(f_{t}\right)$ has zero differential at $\bar{f}$. Instead, $d s_{t}=O_{p}(d t)$ and this drift term is, in fact, negative at $f_{t}=\bar{f}$.

\footnotetext{
${ }^{4}$ The parameter settings for Figure 3 are: $p_{a}=0.3, p_{b}=0.5, p_{c}=1-p_{a}-p_{b}=0.2$; $\lambda_{a}=0.2, \lambda_{b}=0.2 ; \alpha_{b} \in\{1.62,1.64,1.66,1.68\}$, and $\alpha_{a}=1.80$. The decay effect pairs are $\left(\delta_{a}, \delta_{b}\right)=(0.16,014)$ and $\left(\delta_{a}, \delta_{b}\right)=(0.15,0.15)$.
} 
To see this, note that $\bar{f}$ is a local maximum of $s(f)$ so that $s^{\prime}(\bar{f})=0$ and $s^{\prime \prime}(\bar{f})<0$. But, $s^{\prime \prime}(\bar{f})=p_{a} \lambda_{a} \alpha_{a} \sinh \left(\alpha_{a} \bar{f}\right)-p_{b} \lambda_{b} \alpha_{b} \sinh \left(\alpha_{b} \bar{f}\right)$, so that

$$
d s_{t}=\frac{3 \sigma^{2}}{2}\left\{p_{a} \lambda_{a} \alpha_{a} \sinh \left(\alpha_{a} \bar{f}\right)-p_{b} \lambda_{b} \alpha_{b} \sinh \left(\alpha_{b} \bar{f}\right)\right\} d t<0 .
$$

Thus, at the peak of the bubble the local direction of change becomes negative. The collapse of the stochastic asset price process $s_{t}$ begins at the peak and is embodied in the (second order) downward drift of the differential $d s_{t}$ at $f_{t}=\bar{f}$.

The model manifests similar behavior in the case where the parameter configuration involves a market downturn. In that case, $s=s(f)$ still has a turning point at $f=\bar{f}$ but this point now corresponds to a market trough rather than a market peak. Since $s^{\prime}(\bar{f})=0$ and $s^{\prime \prime}(\bar{f})>0$ in this case, we find that (14) is positive rather than negative, so that at the trough $\bar{f}$ we have $d s_{t}>0$. Hence, recovery from the downturn begins at the trough and is embodied in the upward drift of the differential $d s_{t}$.

\section{Modeling Contagion}

The model may be extended to allow for cross market contagion effects. Suppose we have two related markets, such as two real estate markets in different locations or metropolitan districts within the same country. In such cases, we might expect broadly similar fundamentals and regulatory conditions to hold. In particular, if the fundamentals are cointegrated across the two markets, then it will be a reasonable approximation to regard the fundamentals in each case as corresponding to a common stochastic trend in continuous time (c.f., Phillips, 1991)

which we represent as $f_{t}$. One of the markets (perhaps the larger metropolitan area with greater population) might be considered as the core market and the other market a peripheral or secondary market If a speculative bubble arises in one of the markets - say the core market - then interest focuses on possible contagion effects from this market to the other market. Contagion effects can be explored within the framework developed above in the following manner.

We assume that the core market has the specification and equilibrium solution path developed above in (3) and (6). We use the same notation for the variables and parameters that describe this market. The secondary market is assumed to follow the same general specification with the same three classes of investors as before but with possibly different parameterizations and a correspondingly different equilibrium solution. Using the framework above, we write the model and solution for the secondary market using asterisk affixes to distinguish this market from the core market. In particular, employing subjective rational expectations as before we have the following generating mechanism for the two divergent investor types ( $a$ and $b$ ) present in the secondary market

$$
s_{i t}^{*}=f_{t}+\sum_{i=a, b} p_{i}^{*} \gamma_{i}^{*} \mathbb{E}_{i t}\left(d s_{i t}^{*}\right) / d t,
$$


and respective solutions $s_{a}^{*}(f)=f+\frac{\lambda_{i}^{*}}{2 \alpha_{i}^{*}}\left(e^{\alpha_{i}^{*} f}-e^{-\alpha_{i}^{*} f}\right)$, for $i=a, b$ and with $\alpha_{i}=\sqrt{\frac{2}{\gamma_{i}^{*} \sigma^{2}}}$. Aggregating these effects together with the third (fundamentalist) investor type $(c)$ as before, we obtain the solution

$$
s^{*}=f+\frac{p_{a}^{*} \lambda_{a}^{*}}{\alpha_{a}^{*}} \sinh \left(\alpha_{a}^{*} f\right)-\frac{p_{b}^{*} \lambda_{b}^{*}}{\alpha_{b}^{*}} \sinh \left(\alpha_{b}^{*} f\right) .
$$

The exuberance and negativity measures $\lambda_{i}^{*}(i=a, b)$ of the divergent investors in the secondary market are assumed to be based on observed behavior in the core market. In particular, we assume that the $\lambda_{i}^{*}(i=a, b)$ are functionalized ex post to inherit effects of potential contagion from the core market, so that $\lambda_{i}^{*}=\lambda_{i}^{*}(s(f)-f)$ and these secondary market measures of deviation from fundamentals then depend on the extent to which market prices in the core are observed to exceed fundamentals, i.e., $s(f)-f$. Using a linear specification for these inherited effects, we write the dependencies in the following form with no intercept

$$
\lambda_{a}^{*}(f)=\lambda_{a}^{*}(s(f)-f), \quad \lambda_{b}^{*}(f)=\lambda_{b}^{*}(s(f)-f),
$$

where $\lambda_{a}^{*}$ and $\lambda_{b}^{*}$ are fixed slope parameters. The zero intercept in (17) implies that $\lambda_{a}^{*}(f)=\lambda_{b}^{*}(f)=0$ at the origin $f=0$, so that the secondary market follows fundamentals and experiences no exuberant or negative sentiment at the origin, as distinct from the core market where sentiment diverges from fundamentals initially at $f=0$ because $\lambda_{a}, \lambda_{b}>0$ in the core market. With this specification and using $s(f)-f=\frac{p_{a} \lambda_{a}}{\alpha_{a}} \sinh \left(\alpha_{a} f\right)-\frac{p_{b} \lambda_{b}}{\alpha_{b}} \sinh \left(\alpha_{b} f\right)$, the solution for $s^{*}$ takes the following form

$$
\begin{aligned}
s^{*}(f)= & f+\frac{p_{a}^{*} \lambda_{a}^{*}}{\alpha_{a}^{*}}\left[\frac{p_{a} \lambda_{a}}{\alpha_{a}} \sinh \left(\alpha_{a} f\right)-\frac{p_{b} \lambda_{b}}{\alpha_{b}} \sinh \left(\alpha_{b} f\right)\right] \sinh \left(\alpha_{a}^{*} f\right) \\
& -\frac{p_{b}^{*} \lambda_{b}^{*}}{\alpha_{b}^{*}}\left[\frac{p_{a} \lambda_{a}}{\alpha_{a}} \sinh \left(\alpha_{a} f\right)-\frac{p_{b} \lambda_{b}}{\alpha_{b}} \sinh \left(\alpha_{b} f\right)\right] \sinh \left(\alpha_{b}^{*} f\right),
\end{aligned}
$$

which depends on both sets of parameters $\left(\alpha_{a}, \alpha_{b}, p_{a}, p_{b}, \lambda_{a}, \lambda_{b}\right)$ and $\left(\alpha_{a}^{*}, \alpha_{b}^{*}, p_{a}^{*}, p_{b}^{*}, \lambda_{a}^{*}, \lambda_{b}^{*}\right)$, thereby involving both core market and secondary market behavior in the solution path.

Differentiating (18) gives

$$
\begin{aligned}
s^{* \prime}(f)= & 1+\frac{p_{a}^{*} \lambda_{a}^{*}\left(s^{\prime}(f)-1\right)}{\alpha_{a}^{*}} \sinh \left(\alpha_{a}^{*} f\right)-\frac{p_{b}^{*} \lambda_{b}^{*}\left(\left(s^{\prime}(f)-1\right)\right)}{\alpha_{b}^{*}} \sinh \left(\alpha_{b}^{*} f\right) \\
& +p_{a}^{*} \lambda_{a}^{*}(s(f)-f) \cosh \left(\alpha_{a}^{*} f\right)-p_{b}^{*} \lambda_{b}^{*}((s(f)-f)) \cosh \left(\alpha_{b}^{*} f\right),
\end{aligned}
$$

from which it follows that $s^{* \prime}(0)=1$ at $f=0$. Thus, the secondary market follows fundamentals in the vicinity of the origin in contrast to the core market where $s^{\prime}(0)=1+p_{a} \lambda_{a}-p_{b} \lambda_{b}$. While the positive and negative sentiments of core market investors impact the core market immediately, peripheral investor sentiment follows fundamentals at the origin. But when $f>0$, investor sentiment in the core market leads to divergence between the market price and fundamentals 
so that $s(f) \neq f$. This divergence is then transmitted to the secondary market via the functional dependencies $\lambda_{a}^{*}(s(f)-f)$ and $\lambda_{b}^{*}(s(f)-f)$. Since these functions are linear, the exuberance and negativity measures in the secondary market are proportional to the divergence $s(f)-f$ and therefore can be expected to reproduce certain features of the bubble and collapse experienced in the core market with amplification and diminution of these features depending on the magnitude of the divergence $s(f)-f$ and the parametric values of the various coefficients in (18). As such, the model provides a mechanism of direct transmission or contagion from the core to the periphery.

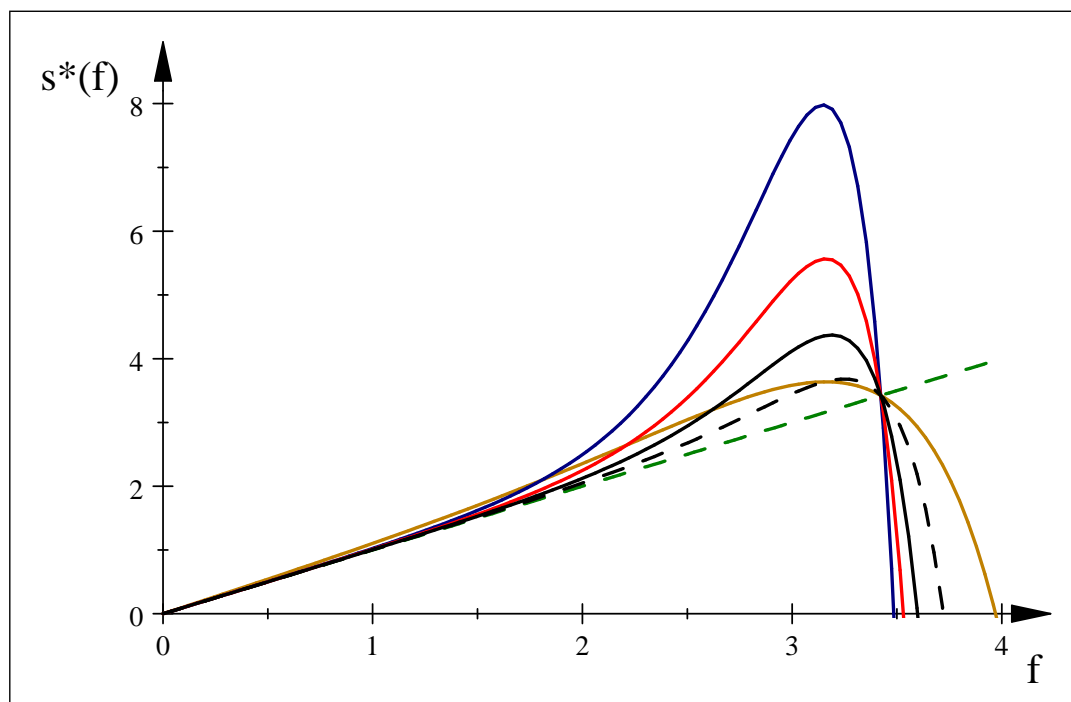

Figure 4: Solution paths $s^{*}(f)$ for equilibrium asset prices in the peripheral market (shown in blue, red, black, black dashed) against the solution path $s(t)$ in the core market. Parameter settings are given in Table 1. The curves are plotted against the $45^{\circ}$ efficient markets line (green dashed) where $s=f$.

Table 1: Parameter Settings for Figure 4

\begin{tabular}{llll}
\hline & Curve Color & Parameters & Values \\
\hline Core: & sienna & $\left(\alpha_{a}, \alpha_{b}, p_{a}, p_{b}, \lambda_{a}, \lambda_{b}\right)$ & $(1.62,1.8,0.5,0.3,0.2,0.2)$ \\
Secondary: & blue & $\left(\alpha_{a}^{*}, \alpha_{b}^{*}, p_{a}^{*}, p_{b}^{*}, \lambda_{a}^{*}, \lambda_{b}^{*}\right)$ & $\left(1.62,1.8,0.5,0.3, \frac{1}{10}, \frac{1}{10}\right)$ \\
& red & $\left(\alpha_{a}^{*}, \alpha_{b}^{*}, p_{a}^{*}, p_{b}^{*}, \lambda_{a}^{*}, \lambda_{b}^{*}\right)$ & $\left(1.62,1.8,0.5,0.3, \frac{1}{20}, \frac{1}{20}\right)$ \\
& black & $\left(\alpha_{a}^{*}, \alpha_{b}^{*}, p_{a}^{*}, p_{b}^{*}, \lambda_{a}^{*}, \lambda_{b}^{*}\right)$ & $\left(1.62,1.8,0.5,0.3, \frac{1}{40}, \frac{1}{40}\right)$ \\
& dashed black & $\left(\alpha_{a}^{*}, \alpha_{b}^{*}, p_{a}^{*}, p_{b}^{*}, \lambda_{a}^{*}, \lambda_{b}^{*}\right)$ & $\left(1.62,1.8,0.5,0.3, \frac{1}{100}, \frac{1}{100}\right)$ \\
Fundamentals & dashed green & & \\
\hline \hline
\end{tabular}

We illustrate the impact of these contagion effects by displaying the equilibrium solution curves $s^{*}(f)$ for the periphery for various parameter values against the corresponding solution curve $s(f)$ for the core market. We use 
the parameter settings given in Table 1 . These settings are the same as those used earlier for the core market and they are replicated here for the secondary market, which is therefore identical in response except for the exuberance and negativity measures for which we use the linear function settings $\lambda_{i}^{*}(s(f)-f)$ with constant slope $\lambda_{i}^{*}$ for $i=a, b$. Thus, in (18), we use the slope settings $\lambda_{i}^{*}=0.10,0.05,0.025$, for both $i=a, b$, and these slopes deliver the secondary market exuberance and negativity departure measures as respective proportions of the core market excess over fundamentals.

Figure 4 shows these solution paths $s^{*}(f)$ for the peripheral market in the blue, red, black, and dashed black curves, against the solution path $s(f)$ for the core market which is given by the sienna curve. The parameter values are given in Table 1. As is apparent in the figure, the core market solution $s(f)$ diverges from fundamentals before the secondary market departs from fundamentals. But once the secondary market inherits sentiment from the core market, the secondary market can be quickly overtaken by contagion. For the blue, red and black parameter settings used in Figure $4\left(\lambda_{i}^{*}=0.1,0.2,0.4 ; i=a, b\right)$, contagion from the core market amplifies exuberance and accelerates the eventual collapse in the periphery. Other settings, such as the dashed black setting $\left(\lambda_{i}^{*}=0.01\right.$; $i=a, b)$ reduce the induced exuberance in the secondary market.

As earlier, we superpose on the components of the model (18) a decay system that achieves long-run recovery returning both the core and periphery markets to fundamental values. The mechanism for the core matches (9) and inhibits both exuberance and negativism in the core in the long run by a decay factor using the functional specifications $p_{a}=p_{a} e^{-\delta_{a} f^{2}}$ and $p_{b}=p_{b} e^{-\delta_{b} f^{2}}$ with $\delta_{a}, \delta_{b} \geq 0$. This mechanism is supplemented with a decay factor for the impact of contagion from the core to the periphery so that $\lambda_{i}^{*}(s(f)-f) \mapsto \lambda_{i}^{*} e^{-\delta_{i}^{*} f^{2}}(s(f)-f)$, which discounts exuberance in the primary market and ensures that contagion effects ultimately die out. The fully modified model that incorporates these decay features has the following explicit form

$$
\begin{aligned}
& s^{*}(f)=f+\frac{p_{a}^{*} \lambda_{a}^{*}}{\alpha_{a}^{*}} e^{-\delta_{a}^{*} f^{2}}\left[\frac{p_{a} \lambda_{a} e^{-\delta_{a} f^{2}}}{\alpha_{a}} \sinh \left(\alpha_{a} f\right)-\frac{p_{b} \lambda_{b} e^{-\delta_{b} f^{2}}}{\alpha_{b}} \sinh \left(\alpha_{b} f\right)\right] \sinh \left(\alpha_{a}^{*} f\right) \\
& -\frac{p_{b}^{*} \lambda_{b}^{*}}{\alpha_{b}^{*}} e^{-\delta_{b}^{*} f^{2}}\left[\frac{p_{a} \lambda_{a} e^{-\delta_{a} f^{2}}}{\alpha_{a}} \sinh \left(\alpha_{a} f\right)-\frac{p_{b} \lambda_{b} e^{-\delta_{b} f^{2}}}{\alpha_{b}} \sinh \left(\alpha_{b} f\right)\right] \sinh \left(\alpha_{b}^{*} f\right) .
\end{aligned}
$$

Figure 5 shows the solution paths $s^{*}(f)$ based on (19) for the parameter settings given in Table 2 . The parameter values closely match those used for Figure 4 but are supplemented with the functionalized long run decay factors $p_{i} e^{-\delta_{i} f^{2}}$ and contagion factors $\lambda_{i}^{*} e^{-\delta_{i}^{*} f^{2}}(s(f)-f)$ for $i=a, b$. The settings for $\left(\delta_{i}, \delta_{i}^{*}\right)_{i=a, b}$ are given in Table 2. As is apparent in the figure from a comparison of the blue and sienna paths with the black and red paths, the decay parameter settings $\left(\delta_{i}, \delta_{i}^{*}\right)_{i=a, b}$ play an important role in the shape of the paths. In particular, small differences in the coefficients $\left(\delta_{i}^{*}\right)_{i=a, b}$ alter the paths from those where the secondary market bubble collapses back to fundamentals (blue and sienna; with $\left.\left(\delta_{a}^{*}, \delta_{b}^{*}\right)=(-0.14,-0.16)\right)$ to those where the secondary mar- 
ket bubble collapses in a downturn market below fundamentals before recovery $\left.\left(\delta_{a}^{*}, \delta_{b}^{*}\right)=(-0.15,-0.16)\right)$. These alternate paths show the impact of small changes in the contagion factors on the equilibrium solution in the secondary market.

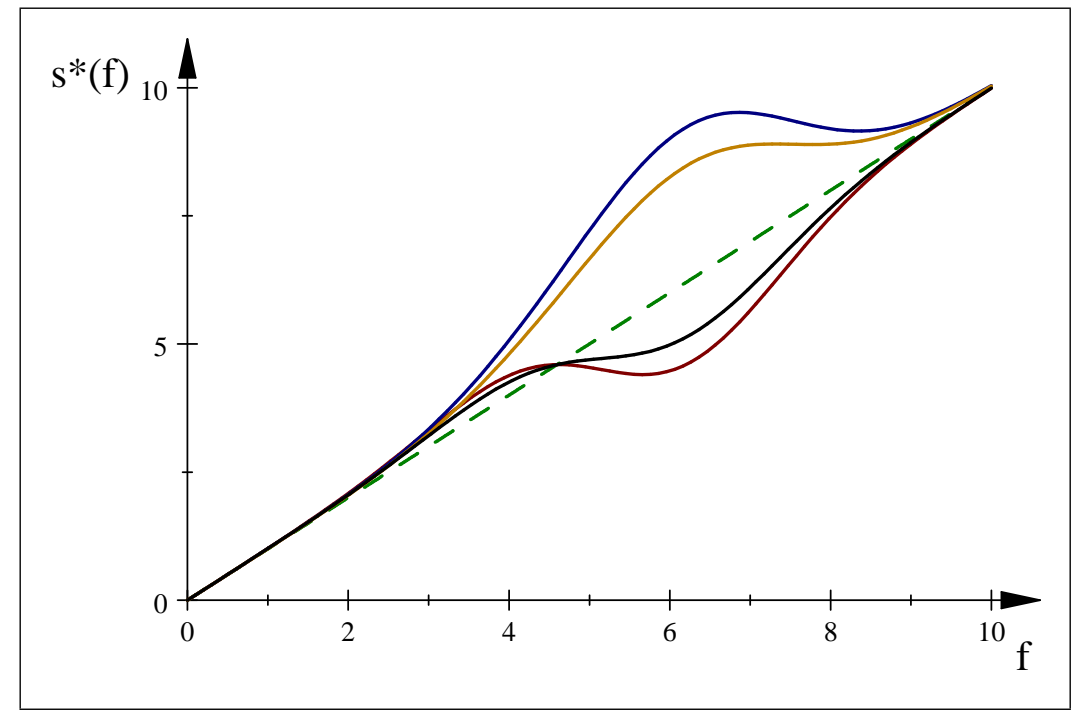

Figure 5: Solution paths for equilibrium asset prices $s^{*}(f)$ in the secondary market allowing for evolutionary decay in the proportions of exuberant and circumspect investors. Parameter values are given in Table 1.

Table 2: Parameter Settings for Figure 5

\begin{tabular}{llll}
\hline & Curve Color & Parameters & Values \\
\hline Core & & $\left(\alpha_{a}, \alpha_{b}, p_{a}, p_{b}, \lambda_{a}, \lambda_{b}\right)$ & $(1.62,1.8,0.5,0.3,0.2,0.2)$ \\
\hline Secondary: & blue & $\left(\alpha_{a}^{*}, \alpha_{b}^{*}, p_{a}^{*}, p_{b}^{*}, \lambda_{a}^{*}, \lambda_{b}^{*}\right)$ & $\left(1.62,1.8,0.5,0.3, \frac{1}{30}, \frac{1}{30}\right)$ \\
& sienna & $\left(\alpha_{a}^{*}, \alpha_{b}^{*}, p_{a}^{*}, p_{b}^{*}, \lambda_{a}^{*}, \lambda_{b}^{*}\right)$ & $\left(1.62,1.8,0.5,0.3, \frac{1}{40}, \frac{1}{40}\right)$ \\
& black & $\left(\alpha_{a}^{*}, \alpha_{b}^{*}, p_{a}^{*}, p_{b}^{*}, \lambda_{a}^{*}, \lambda_{b}^{*}\right)$ & $\left(1.62,1.8,0.5,0.3, \frac{1}{30}, \frac{1}{30}\right)$ \\
& red & $\left(\alpha_{a}^{*}, \alpha_{b}^{*}, p_{a}^{*}, p_{b}^{*}, \lambda_{a}^{*}, \lambda_{b}^{*}\right)$ & $\left(1.62,1.8,0.5,0.3, \frac{1}{20}, \frac{1}{20}\right)$ \\
Decay factor & blue \& sienna & $\left(\delta_{a}, \delta_{b}\right),\left(\delta_{a}^{*}, \delta_{b}^{*}\right)$ & $(-0.15,-0.15),(-0.14,-0.16)$ \\
& black \& red & $\left(\delta_{a}, \delta_{b}\right),\left(\delta_{a}^{*}, \delta_{b}^{*}\right)$ & $(-0.15,-0.15),(-0.15,-0.16)$ \\
Fundamentals & dashed green & & \\
\hline \hline
\end{tabular}

More generally, this example demonstrate the fragility of the bubble expansion and collapse paths to small changes in the model parameters. There is a good reason why such fragility might be anticipated. The fragility of the shapes of these solution paths may be taken as indicative of a well-known empirical phenomena concerning bubbles. Real world bubbles share many common characteristics of speculative expansion followed by contraction. But each new 
bubble nonetheless appears different from past bubbles, justifying the appellation that "this time is different" and thereby necessitating flexibility in the range of possible solution paths if a theory model is to be useful in practical work.

\section{Empirical Model Implications}

From (13), the local behavior of the price process following the point of origination is governed by a linear diffusion. The solution of this equation is given by $s_{t}=\int_{0}^{t} e^{\beta(t-r)} d f_{r}$ and discrete time observations $\left(s_{k h_{n}}\right)_{k=0}^{K}$ taken at the sampling interval $h_{n}$ (where $n$ is the number of sample observations) are known to follow the first order autoregression (AR)

$$
s_{k h_{n}}=\rho_{n} s_{k h_{n}-h_{n}}+u_{k h_{n}}, \text { with } \rho_{n}=e^{\beta h_{n}} \text { and } u_{k h_{n}}=\int_{k h_{n}-h_{n}}^{k h_{n}} e^{\beta\left(k h_{n}-r\right)} d f_{r},
$$

(see Phillips, 1972). This autoregression (20) is (mildly) explosive when the O-U coefficient $\beta>0$ and the sampling interval $h_{n}=\frac{1}{k_{n}}$ for some sequence $k_{n} \rightarrow \infty$ for which $\frac{k_{n}}{n} \rightarrow 0$. In such cases the AR coefficient $\rho_{n} \sim 1+\frac{\beta}{k_{n}}>1$ and the autoregression is said to be mildly explosive in the terminology of Phillips and Magdalinos (2007). When $\beta<0$, the AR coefficient is $\rho_{n}=1+\frac{\beta}{k_{n}}<1$ and is called mildly integrated.

Mildly explosive AR processes of the form (20) have been used as empirical models of bubble behavior in several recent articles (Phillips, Wu and $\mathrm{Yu}, 2011$; Phillips and Yu, 2011; Phillips, Shi and Yu, 2015a, 2015b). In this empirical work, the model (20) is treated as a reduced form and is used to test for the existence of bubbles in financial, commodity, and real estate asset prices by means of right-sided unit root tests of the hypothesis $\mathbb{H}_{0}: \rho_{n}=1$ in (20) or $\beta=0$ in (13). $\mathbb{H}_{0}$ is used to represent martingale behavior under market normalcy corresponding to $d s_{t}=\omega d f_{t}$ in (13), as distinct from mildly explosive behavior of $s_{t}$ under the alternative hypothesis $\mathbb{H}_{1}: \rho_{n} \sim 1+\frac{\beta}{k_{n}}>1$, or $\beta>0$ in (13).

The present work shows that such reduced form models can be justified in terms of a structural model based on subjective rational expectations of market opportunities and the aggregation of diverse investor assessments of the impact of fundamentals in determining market prices. In other recent work (Phillips and Shi, 2014), a mildly integrated AR process has been used as a reduced form to model the bubble collapse process. Again, this formulation may be justified using the present rational expectations framework.

In addition to testing and dating bubbles, the framework may be used to assess empirical evidence of contagion between two markets. In practical empirical work it is most convenient again to work with the local specifications in terms of linear diffusions and focus on linkages between the implied parameters $\beta$ and $\beta^{*}$ of the core and secondary models in continuous time with the associated AR parametric specifications $\rho_{n}$ and $\rho_{n}^{*}$ relating to discrete time ob- 
servations in the core and secondary markets. Empirical evidence of contagion can be explored empirically by examining linkages between these parameters over time when they are estimated by a recursive or moving window approach through the sample. In recent work, Greenaway-McGrevy and Phillips (2016) provide an empirical illustration of this linkage technique in detecting contagion between metropolitan real estate markets. The econometric methods used in fitting the recursive relationship between the AR coefficients involves a time varying coefficient model, which allows the relationship between $\rho_{n}$ and $\rho_{n}^{*}$ to vary over time and which can be estimated by kernel regression methods.

\section{Conclusion}

The model developed in this paper has a simple stylized form involving only three types of investors, working with their own assessments of the impact of fundamentals on prices via a subjective rational expectations framework. The model uses an aggregative mechanism to determine market price and does not attempt to capture the trading mechanism through which these diverse market assessments coalesce and work to determine market prices. From this perspective, the present model offers only a plausible behavioral mechanism of price determination.

Despite its simplicity, the model is capable of capturing some of the major features of financial and real estate markets that manifest bubble-like behavior involving exuberance and collapse. Multi-market versions of the model also accommodate cross-market contagion of speculative and negative market sentiment about the impact of fundamentals. Local approximations show that the model is compatible with reduced form autoregressive specifications that are now being used to provide empirical tests for the existence of bubbles in financial and real estate markets, as well as dating algorithms that can consistently estimate origination and termination dates of bubble activity. These methods are used recursively and, in so doing, are able to provide mechanisms for real time assessments of financial market conditions by surveillance teams in central banks. The present model helps to furnish support for the use of these reduced form specifications in empirical work and to interpret their findings in terms of the impact of investor sentiment and expectations.

One feature to emerge from the workings of the model is the fragility of the solution paths to certain parameter settings. This fragility is partly explained by the fact that the general solution involves exponential departures from fundamentals that may be accentuated or attenuated depending on the magnitude of the exuberance and negativity measures, the proportions of investors of each type, and any incoming contagion effects from another market. The fragility of the shapes of these solution paths may be taken as indicative of a well-known empirical phenomena concerning bubbles. Real world bubbles share many common characteristics of speculative expansion followed by contraction. But each new bubble nonetheless appears different from past bubbles, justifying the appellation that "this time is different" and thereby necessitating flexibility in the 
range of possible solution paths if a theory model is to be useful in practical work.

\section{$7 \quad$ References}

Abreu, D. and Brunnermeier, M. K., (2003). Bubbles and crashes, Econometrica, 71, 173-204.

Akerlof, G. A. and Shiller, R. J., (2009). Animal Spirits: how human psychology drives the economy and why it matters for global capitalism. Princeton: Princeton University Press.

Becker, R. A. (2008). Transversality condition, in S. N. Durlauf and L. E. Blume (Eds.), The New Palgrave Dictionary of Economics, Second Edition, 2008, Palgrave.

Bertola, G. (1998). Irreversible investment. Research in Economics, 52, 3-37.

Harrison J. M. and D. Kreps., (1978). Speculative investor behavior in a stock market with heterogeneous expectations. Quarterly Journal of Economics, 89, 323-336.

Dixit, A. (1993). The Art of Smooth Pasting. Chur: Harwood Academic Publishers.

Greenaway-McGrevy, R. and P. C. B. Phillips, (2016). "Hot Property in New Zealand: Empirical Evidence of Housing Bubbles in the Metropolitan Centres", New Zealand Economic Papers (forthcoming).

Keynes, J. M. (1936). The General Theory of Employment Interest and Money. Macmillan, London.

Krugman, P. (1991). "Target Zones and Exchange Rate Dynamics", Quarterly Journal of Economics, Vol. 106. 669-682.

Krugman, P. and M. Miller (1992). Exchange Rate Targets and Currency Bands. Cambridge: Cambridge University Press.

Lee, J-H. and P. C. B. Phillips (2016). "Asset Pricing with Financial Bubble Risk", Journal of Empirical Finance (forthcoming).

Li, W. and Xue, H., 2009, A Bayesian's Bubble. The Journal of Finance, 64(6), 2665-2701.

Liang, J., P. C. B. Phillips and J. Yu (2016). "A New Hedonic Regression for Real Estate Prices Applied to the Singapore Residential Market" Journal of Banking and Finance, 2016 (forthcoming).

Phillips, P. C. B. (1972). "The Structural Estimation of a Stochastic Differential Equation System," Econometrica 40:6, 1021-1041. 
Phillips, P. C. B. (1991). "Error Correction and Long Run Equilibria in Continuous Time," Econometrica 59, 967-980.

Phillips, P. C. B., T. Magdalinos (2007a). "Limit Theory for Moderate Deviations from a Unit Root," Journal of Econometrics, 136, 115-130.

Phillips, P. C. B. and S. Shi (2014) "Financial Bubble Implosion", Cowles Foundation Discussion Paper, Yale University.

Phillips, P.C.B., S. Shi, and Y. Yu (2015a). "Testing for Multiple Bubbles: Historical episodes of exuberance and collapse in the S\&P 500 ", International Economic Review, 56, 1042-1076.

Phillips, P.C.B., S. Shi, and Y. Yu (2015b). "Testing for Multiple Bubbles: Limit theory of Real Time Detectors", International Economic Review, $56,1077-1131$.

Phillips P. C. B., Y. Wu and J. Yu (2011). "Explosive behavior in the 1990s Nasdaq: When did exuberance escalate asset values?", International Economic Review, 52, 201-226.

Phillips P. C. B. and J. Yu (2011). "Dating the Timeline of Financial Bubbles during the Subprime Crisis," Quantitative Economics, 2, pp. 455-491.

Scheinkman, J. and W. Xiong, (2003). Overconfidence and speculative bubbles, Journal of Political Economy, 111, 1183-1219. 